\title{
Displasia cleidocraniana: um estudo de caso
}

\author{
Cleidocranial dysplasia: a case study
}

Displasia cleidocraneal: un estudio de caso

Bianca Sobral Bellemo ${ }^{1 \star}$, Thiago Alves Hungaro', Ana Carolina Zanin Sacoman Kurihara.

\begin{abstract}
RESUMO
Objetivo: relatar caso de Displasia Cleidocraniana no intuito de auxiliar o diagnóstico precoce e prognóstico dos pacientes acometidos. Detalhamento do caso: criança do sexo masculino nascida a termo, realizado 10 consultas de acompanhamento pré-natal. Pai, mãe e irmão hígidos. Após o parto foi realizado o exame físico do recém-nascido e não foi possível palpar as clavículas e notou-se um alargamento das fontanelas. Sendo encaminhado para a radiografia, evidenciando-se apenas o terço proximal das clavículas direita e esquerda ossificados, não sendo possível avaliar o terço médio e distal, tampouco o núcleo secundário de ossificação do úmero e processo coracóide da escápula bilateralmente. Ademais, observou-se redução da mineralização óssea da calota craniana com alargamento das fontanelas. Considerações finais: A Displasia Cleidocraniana (DCC) é uma doença hereditária autossômica dominante ocasionada por uma mutação no gene RUNX2, fator esse que codifica a transcrição para o osteoblasto, e é essencial para a diferenciação celular dos osteoblastos e odontoblastos que são fundamentais para a síntese e metabolismo do tecido ósseo. Por se tratar de uma anomalia genética rara, quanto mais precoce for feito o diagnóstico, mais eficaz será o acompanhamento da equipe multidisciplinar no atendimento diante das necessidades do portador da DCC e da sua família.
\end{abstract}

Palavras-chave: Displasia cleidocraniana, Diagnóstico precoce, Dente supranumerário.

\begin{abstract}
Objective: To report a case of Cleidocranial Dysplasia in order to help early diagnosis and prognosis of affected patients. Case Detail: full-term male child, who underwent 10 prenatal follow-up appointments. Healthy father, mother and brother. After delivery, the newborn was physically examined and it was not possible to palpate the clavicles and widen the fontanelles. He was referred for radiography, showing only the proximal third of the ossified right and left clavicles, and it was not possible to evaluate the middle and distal third, nor the secondary nucleus of humeral ossification and the scapular coracoid process bilaterally. In addition, there was a reduction in bone mineralization of the skullcap with widening of the fontanelles. Final considerations: Cleidocranial dysplasia (CHD) is an autosomal dominant inherited disease caused by a mutation in the RUNX2 gene, which encodes transcription to osteoblasts, and is essential for the cellular differentiation of osteoblasts and odontoblasts that are fundamental for the synthesis and bone tissue metabolism. Because it is a rare genetic anomaly, the earlier the diagnosis is made, the more effective will be the monitoring of the multidisciplinary team in meeting the needs of the patient with CHD and his family.
\end{abstract}

Keywords: Cleidocranial dysplasia, Early diagnosis, Supernumerary teeth.

\section{RESUMEN}

Objetivo: Reportar un caso de displasia cleidocraneal con el objetivo de ayudar a su diagnóstico temprano y prognosis de los pacientes afectados. Detalle del caso: infante masculino nacido a termino completo, quién tuvo 10 citas prenatales de seguimiento. Padre, madre y hermano sanos. Posterior al parto el recién nacido

${ }^{1}$ Centro Universitário Municipal de Franca (Uni-FACEF), Franca - SP.

*E-mail:biancasbellemo@gmail.com

SUBMETIDO EM: 2/2020 
fue examinado físicamente y no fue posible palpar las clavículas y extender las fontanelas. Fue enviado a radiografía, mostrando solamente una tercera parte aproximadamente Fue derivado para radiografía de las clavículas izquierda y derecha osificada y no fue posible evaluar el medio y tercer distal, así como el núcleo secundario de la osificación del húmero y el proceso escapular coracoide bilateralmente. En adición, hubo una reducción en la mineralización de la escutelaria con un ensanchamiento de las fontanelas. Consideraciones finales: la displasia cleidocraneal (chd) es una enfermedad autosómica dominante hereditaria causada por una mutación en el gen RUNX2, El cual codifica las transcripciones a osteoblastos, y es esencial para la diferenciación celular de los osteoblastos y odontoblastos que son fundamentales para la síntesis y el metabolismo del tejido óseo. Debido a que es una inusual anomalía genética, entre más pronto se realice el diagnóstico será más efectivo el monitoreo del equipo multidisciplinario en atender las necesidades del paciente con chd y sus familiares.

Palabras clave: Cleidocraneal displasia, Diagnóstico temprano, Diente supernumerario.

\section{INTRODUÇÃO}

A Displasia Cleidocraniana (DCC), também conhecida como Disostose Cleidocraniana, é um distúrbio genético raro, sem predileção de sexo ou raça, com uma prevalência entre 1:200.000a 1:1000.000. (PORCIUNCULA CGG, et al., 2013; MACHADO CV, et al., 2010).É uma doença hereditária autossômica dominante ocasionada por uma mutação no gene RUNX2 (Runt-Related Transcription Factor 2)(HARDY A, et al., 2015).

Esse fator codifica a transcrição para o osteoblasto, sendo essencial para a diferenciação celular dos osteoblastos e odontoblastos que são fundamentais para a síntese e metabolismo do tecido ósseo. Sendo assim, os pacientes portadores da síndrome apresentam disfunções em seu sistema esquelético (TRINDADE AKF, et al., 2010; CHEN S, et al., 2005). Segundo Silva Junior NA, et al (2007), ainda diante desta disfunção é comumente observado que os portadores de DCC apresentem alterações como: fontanelas largas, retardo no desenvolvimento esquelético do crânio, cabeça braquiocefálica, hipertelorismo, prognatismo mandibular, palato estreito e profundo, sinais de baixa estatura, e excessiva largura no pescoço.

Todavia, apesar de todas essas alterações descritas, Queiroz RM, et al. (2017) pontua que é importante ressaltar que o diagnóstico é baseado na tríade patognomônica que se observa clinicamente pela presença de suturas cranianas persistentemente abertas, agenesia clavicular bilateral ( $80 \%$ dos casos) e por último, dentes supranumerários, também conhecido como hiperdontia (93-100\%). A constatação diagnóstica acontece pela presença sintomatológica desta tríade clínica e com sua confirmação radiológica.

Outro ponto importante a ser ressaltado na investigação segundo Karagüzel G, et al. (2010), é realizar o diagnóstico diferencial com a patologia conhecida como Picnodisostose, que apresenta características clínicas muito semelhantes a DCC, como por exemplo a fragilidade óssea, a osteoclerose, o atraso no fechamento das suturas cranianas, a baixa estatura, anotondia e atraso na erupção dos dentes permanentes. Entretanto, segundo autores como Desmyttere M, et al. (2017) e Tanaka JL, et al. (2006), a presença de osteoclerose e a ausência de dentes supranumerarios já são suficientes para diferenciar as patologias.

Portanto este artigo tem como objetivo relatar um caso de Displasia Cleidocraniana em um recém-nascido (RN), recebido por uma equipe médica de um hospital do interior de São Paulo, buscando ampliar a divulgação do conhecimento sobre esta patologia rara, enfatizando os meios diagnósticos precoces, facilitando o acesso aos profissionais sobre o reconhecimento da doença, implicando diretamente na qualidade de vida destes pacientes e sua assistência às necessidades de saúde.

\section{DETALHAMENTO DO CASO}

Gestante G. S. A. P., 30 anos, natural de Orlândia/SP, com história obstétrica de 01 (uma) gestação anterior, resolvida através de um parto do tipo cesárea, deu entrada no Pronto Atendimento do Hospital São Marcos, no município de Morro Agudo/São Paulo, com 39 semanas de gestação. 
O pré-natal foi iniciado no $1^{0}$ mês de gestação, composto por 10 (dez) consultas subsequentes, apresentando data da última menstruação (DUM): 25/05/2017, com sorologias negativas, tipagem sanguínea A negativo e demais resultados de exames laboratoriais, relativos às baterias de screening do pré-natal, sem quaisquer alterações.

Foram realizadas três ultrassonografias obstétricas ao longo da gestação, dentre elas um ultrassom morfológico durante o segundo trimestre gestacional, sendo que nenhum dos exames de imagem revelou alterações no feto.

A última consulta do pré-natal ocorreu dia 14/02/2018 sem intercorrências, sendo avaliada a ultrassonografia obstétrica realizada em 10/01/2018. A mesma evidenciava feto único em situação longitudinal com apresentação cefálica e dorso à esquerda; líquido amniótico com volume normal; diâmetro Bi-parietal 8,0 $\mathrm{cm}$; circunferência craniana $29,5 \mathrm{~cm}$; circunferência abdominal $29,2 \mathrm{~cm}$; comprimento do fêmur $5,9 \mathrm{~cm}$ e peso fetal $1970 \mathrm{~g}+/-10 \%$.

A cesariana aconteceu sem intercorrências aos 20/02/2018, pela manhã, conforme previamente programada, resultando em recém-nascido do sexo masculino; peso de $3.045 \mathrm{~g}$; estatura $47,5 \mathrm{~cm}$; perímetro cefálico (PC) $32,5 \mathrm{~cm}$; perímetro torácico (PT) $33,5 \mathrm{~cm}$. O RN apresentou Apgar de primeiro minuto: 10, mantido no quinto e décimo minuto.

Conforme protocolo foi realizada a coleta da tipagem sanguínea: B+. Durante o exame físico, na recepção do $\mathrm{RN}$ pelo pediatra, não foi possível palpar as duas clavículas, quando foi determinada a conduta da realização de radiografias de crânio, tórax e membros inferiores, sendo o mesmo encaminhado para realização dos exames complementares.

Durante a avaliação da radiografia de crânio foram detectadas: ausência de calcificação de calota craniana na região sagital de alta convexidade; ausência de calcificações patológicas intracranianas e sela túrcica normal (Figura 1).

A análise da radiografia de tórax, permitiu a avaliação das clavículas, onde foram apenas identificados o terço proximal das clavículas direita e esquerda ossificados, não sendo possível avaliar o terço médio e distal, tampouco, o núcleo secundário de ossificação do úmero e processo coracóide da escápula bilateralmente, além de aumento de área cardíaca com desvio mediastinal para a direita (Figura 2).

Figura 1 - Radiografia de crânio AP+P.

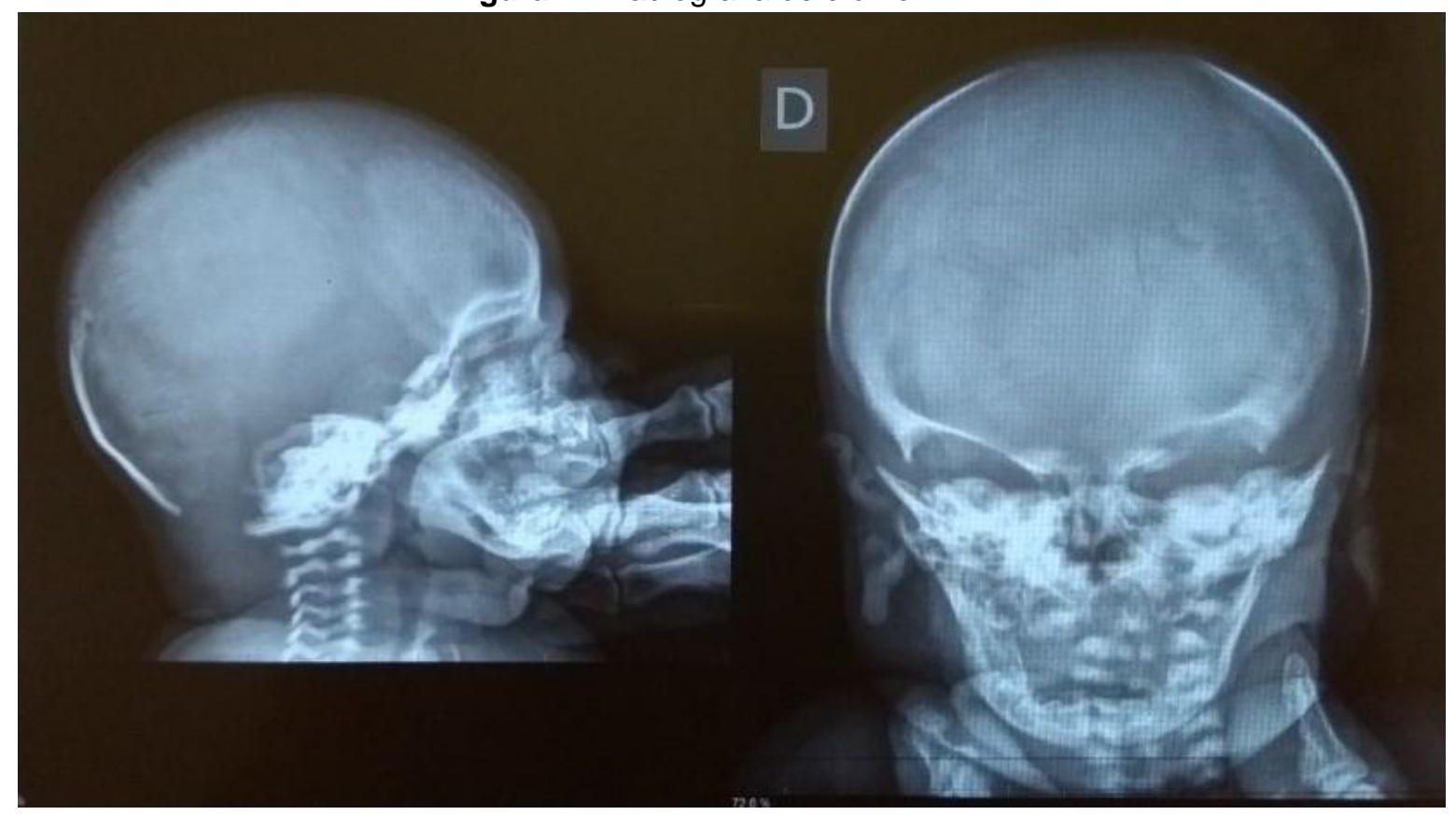

Fonte: Bellemo BS, et al., 2019 
Figura 2 - Radiografia do Tórax PA, braços direito e esquerdo.

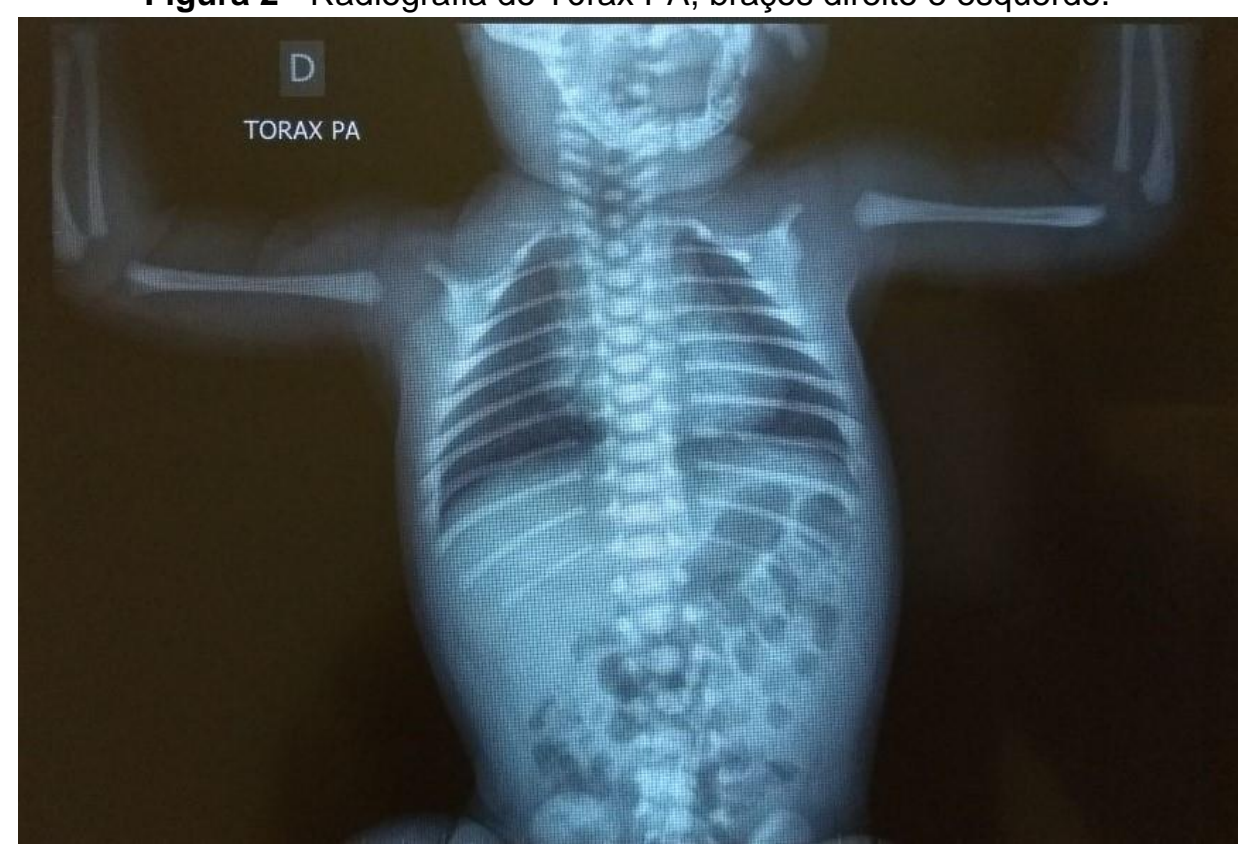

Fonte: Bellemo BS, et al., 2019.

Fica evidente, após a observação das imagens radiográficas, a ausência dos núcleos de ossificação proximais dos úmeros (Figura 2), bem como a redução da mineralização óssea da calota craniana com alargamento das fontanelas, em comparação com o esperado para um RN a termo (Figura 1). Tais achados sugerem retardo na maturação óssea, apesar textura óssea normal compatível com a idade gestacional de um $\mathrm{RN}$ a termo, com gestação resolvida com 39 semanas.

Também foram realizadas radiografia das pernas direita e esquerda (fêmur), como mostra a Figura 3 onde foi possível constatar ausência dos núcleos de ossificação proximais e distais do fêmur bilateralmente, e ausência dos núcleos de ossificação proximais das tíbias comumente presentes no RN a termo, reforçando o diagnóstico de retardo na maturação óssea visualizado previamente nas demais radiografias.

Figura 3 - Radiografia de ossos longos - Pernas direita e esquerda.

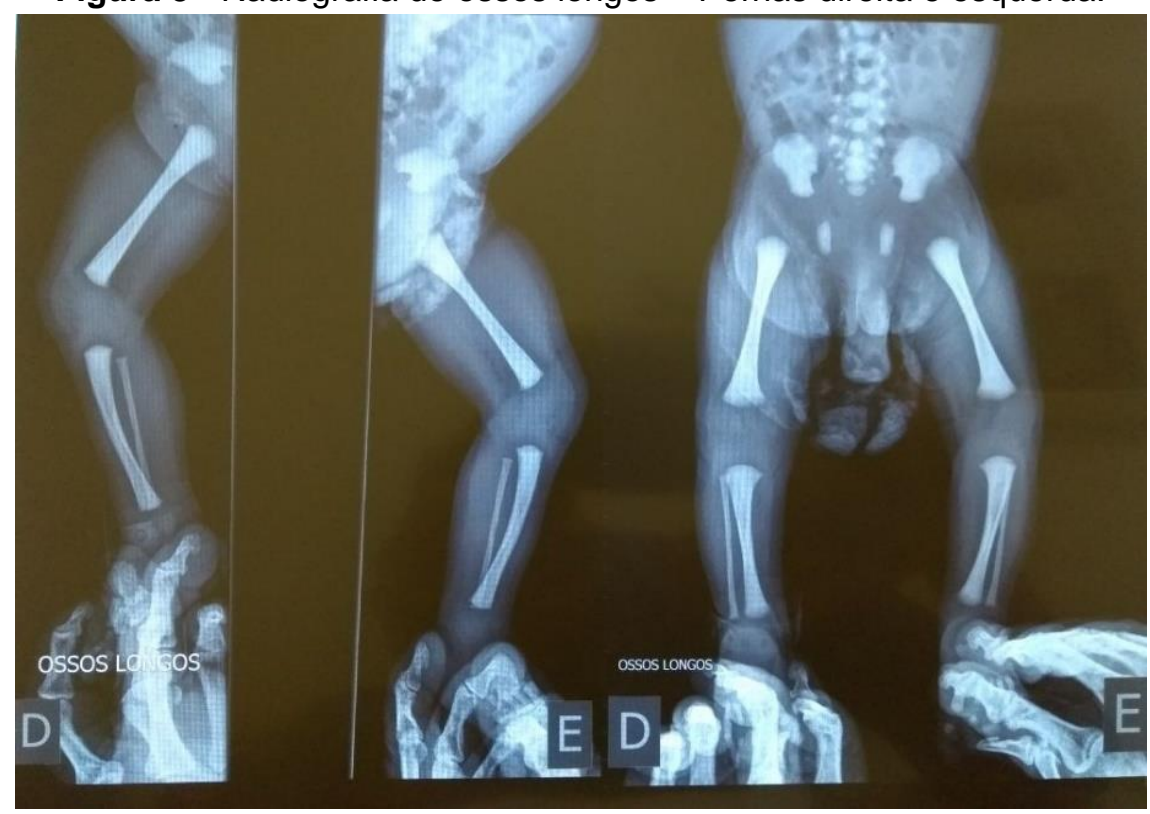

Fonte: Bellemo BS, et al., 2019. 
O quadro clínico observado no paciente deste relato, associado aos achados de imagem radiológicos, permitem forte suspeição relacionada a patologia de atraso do desenvolvimento ósseo, compatível com Disostose Cleidocraniana, sendo necessária avaliação dentária e avaliação genética para confirmação da hipótese diagnóstica. Para tal, após a alta hospitalar, o paciente foi referenciado para avaliação odontológica e para o serviço terciário em ambulatório de genética médica, no Hospital das Clínicas da Faculdade de Medicina da USP (FMUSP), onde mantém acompanhamento regular com equipe multiprofissional, permitindo o seguimento adequado relativo à patologia, assim como, melhor qualidade de vida e saúde ao paciente.

\section{DISCUSSÃO}

O exame físico detalhado do RN após o parto, assim como os exames radiológicos realizados ajudaram no diagnóstico precoce da DCC, proporcionando acompanhamento integral e multidisciplinar para a criança e sua família, que vai ao encontro dos artigos dos autores GASSEN HT et al.( 2006); SERRATINE ACP e ROCHA R (2007). Cabe pontuar que na maioria dos casos a descoberta da DCC acontece tardiamente por profissionais dentistas, devido as anomalias dentárias o que dificulta o tratamento e a qualidade de vida dessas pessoas (PAN CY et al, 2017).

Segundo os estudos dos autores Schnutenhaus S et al (2015) e Avendaño A et al (2018), o tratamento é mais efetivo quando iniciado na infância ou adolescência, alcançando maior possibilidade de tornar-se bemsucedido, principalmente do ponto de vista odontológico, pois, proporciona resolução mais simples e com custo otimizado, resultando em melhorias na qualidade de vida desse paciente. É comum como mostra a literatura que ocorram nessa fase da infância a remoção dos dentes temporários e potenciais obstáculos para a correta erupção dentária permanente (HAESE K e TOUX GL, 2020).

Cabe lembrar que apesar de todo tratamento odontológico e ortodôntico, muitas vezes, ainda podem persistir deformidades estéticas, uma vez que esses indivíduos apresentam desproporcionalidade entre cabeça e o restante do corpo, uma ampla base do nariz com uma ponte nasal deprimida, e geralmente apresentam aspecto de prognata devido ao crescimento deficiente da maxila. que acabam por interferir na aceitação da autoimagem desse paciente. (SANTOS RLO, et al 2016). Assim sendo, a presença do profissional da psicologia enriquece o trabalho dessa equipe multidisciplinar, pois os pacientes portadores de DCC requisitam acompanhamento por diversos profissionais, das diferentes áreas, afim de ampliar o acesso aos diversos tratamentos demandados, assim como, resolução das sequelas de maneira conjunta, permitindo adequação das mesmas as necessidades reais do paciente e seu desenvolvimento. (SCHNUTENHAUS $S$ et al, 2015).

Segundo o estudo do Haese K e Toux GL, (2020) o aconselhamento genético deve ser fornecido sempre que houver suspeitas sobre a DCC , porém apesar de estar claro que se trata de uma anomalia genética com alto risco de recorrência, o paciente deste relato não apresentava nenhum antecedente direto com história de DCC, justificando ainda mais a importância de reforçar as orientações junto à família em relação à necessidade de uma de investigação e acompanhamento genético (ARAYA BI et al, 2013).

Considerando os dados obtidos durante a avaliação/exame mais recente do paciente, realizada aos 16 meses de idade, foi possível constatar que sua estatura encontra-se no escore- $Z$ - 3 do acompanhamento para crescimento em meninos (o que revela que o mesmo se encontra distante da mediana da referência para a idade, altura e sexo, ou seja, -3 é considerado uma baixa estatura proporcional) (BRASIL,2012). Ainda segundo Kolokitha OE e loannidou L (2013) é permitido afirmar que a baixa estatura é certamente um dos aspectos característicos relacionados aos pacientes portadores de DCC. Porém apesar de ao nascimento a criança apresentar comprimento adequado, ela apresentará esse déficit no crescimento ao longo do seu desenvolvimento, podendo chegar em média 165 centímetros (+/- 8cm) (MA Y, ZHAO F e YU D, 2019).

Durante o exame clínico, ao segurar as mãos do paciente cruzadas anteriormente ao tórax com os braços esticados, foi observado aproximação excessiva entre os ombros na região anteromedial. Essa manobra é positiva quando ocorre agenesia total ou parcial bilateral das clavículas e é percebida em 93-99\% dos portadores de DCC, conforme descrito por Queiroz RM et al (2017). Foi ainda percebido que apesar de apresentar significativo atraso do fechamento da fontanela bregmática $(5,5 \mathrm{~cm} \times 5,5 \mathrm{~cm})$, a criança não 
apresentou indícios de atrasos do desenvolvimento neuropsicomotor, realizando sinais e atitudes condizentes com o desenvolvimento normal, sendo capaz de andar sem apoio, pegar e erguer objetos, responder a comandos familiares e de desconhecidos, mantendo o quadro do desenvolvimento neurológico e reflexos neurológicos sem alterações, como evidenciam as Diretrizes de Estimulação Precoce: crianças de 0 a 3 anos com atraso no desenvolvimento psicomotor do Ministério da Saúde (2006). Na avaliação do desenvolvimento dentário pode-se observar atraso da primeira dentição e também da definitiva, de acordo com Araya BI et al (2013) ou dentes supranumerários e impactados. No paciente em questão a dentição surgiu dentro do prazo previsto e não foi solicitado radiografia panorâmica para melhor avaliação. É importante ressaltar que a indicação dessa radiografia possibilita o planejamento de intervenções odontológicas futuras e preventivas na criança (TRINDADE AKF et al, 2010).

A DCC é uma patologia rara, que acarreta importantes repercussões clínicas e na qualidade de vida para o portador e seus familiares, ademais, gera custos para o sistema público para realização de seu seguimento. Diante disso, é importante fortalecer a necessidade do rastreio desta patologia o mais precoce possível como ocorrido no caso apresentado, divergindo da maioria dos artigos da revisão bibliográfica que ampara esse estudo, onde foi observado um diagnóstico tardio. (GASSEN HT, et al., 2006; KARAGÜZEL G, et al., 2010; KOLOKITHA OE e IOANNIDOUL, 2013; MACHADO CV, et al., 2010; PORCIUNCULA CGG, et al.,2013; QUEIROZ RM, et al., 2017; SCHNUTENHAUS S, et al., 2015; SERRATINE ACP e ROCHA R ,2007; SILVA JÚNIOR AN, et al., 2007; TANAKA JL, et al.,2006; TRINDADE AKF, et al., 2010).

Portanto, após realização da revisão de literatura e sua correlação com o relato de experiência relativo ao paciente da descrição, é possível concluir que para realização do diagnóstico precoce desta patologia, devese avaliar alterações anatômicas do concepto, através de exame físico criterioso na recepção pós parto, assim como análise das informações e história da herança genética familiar, que pode ser obtida através de anamneses completas durante as consultas de pré-natal. Estes pesquisadores reforçam, que os impactos financeiros na aplicação deste rastreio seriam mínimos, tendo em vista que ambos compõem itens a serem observados na rotina do pré-natal normal e durante o exame físico detalhado do RN após o parto, não resultando, dessa forma, em incrementos da receita relativos a este processo diagnóstico.

Os pesquisadores entendem ao término deste relato de experiência, que existe a necessidade de proposição relacionada a criação de novas políticas de saúde pública, voltadas para o seguimento dos portadores desta patologia, garantindo-lhes a realização de diagnósticos precoces e permitindo que durante o seu desenvolvimento o paciente realize acompanhamento com as especialidades essenciais, relacionadas à DCC, assim como promover o fortalecimento dos grupos de apoio para os portadores e seus familiares, incrementando vínculo e sua rede de apoio. A ampliação do acesso ao conhecimento de patologias raras, permite aos profissionais médicos e da equipe de saúde, assim como para a população em geral, que se disseminem informações que outrora eram mantidas apenas nos grandes centros de especialidades, o que restringia o diagnóstico da patologia e prognóstico dos pacientes acometidos. Portanto, este relato de experiência, proporciona que a comunidade acadêmica e profissionais de saúde, reconheçam precocemente as deficiências das suas ações no reconhecimento da patologia, durante o pré e pós-natal, assim como consigam realizar melhor acompanhamento do paciente portador de DCC, sendo que, tais ações implicarão positivamente na qualidade de vida e indicadores de saúde relacionados a esta população.

\section{REFERÊNCIAS}

1. ARAYA BI, et al. Caso clínico-radiológico para diagnóstico. Revista. chilena. pediatria, Santiago, $2013 ; 84$ (5); 550553

2. AVENDAÑO A, et al. Cleidocranial dysplasia. A molecular and clinical review. International Dental Research 2018; $8(1): X-X$.

3. BRASIL- Ministério da Saúde. Secretaria de Atenção à Saúde. Diretrizes de Estimulação Precoce: crianças de 0 a 3 anos com atraso no desenvolvimento psicomotor. Brasilia: Ministério da Saúde; 2006

4. BRASIL-Ministério da Saúde. Secretaria de Atenção à Saúde. Departamento de Atenção Básica. Saúde da criança: Crescimento e Desenvolvimento. Brasília: Cadernos de Atenção básica, 33 pg 110, 2012

5. CHEN S, et al. Altered gene expression in human cleido cranial dysplasia dental pulp cells. Archives of Oral Biology, 2005; 50(2):227-236 
6. DESMYTTERE M, et al. Oral management of two sisters with pycnodysostosis. Med Buccale Chir Buccale 2017; 23:139-145

7. GASSEN HT,et al. Relato de dois casos familiares de disostose Cleidocraniana. Revista Faculdade de Odontologia UFP. 2006; 11:31-5.

8. HAESE K; TOUX GL. Surgical Strategy for patients with late-diagnosed minor form of Cleidocranial Dysplasia: three cases. 2020. Journal of Oral Medicine and Oral Surgery. 26:4

9. HARDY A, et al. Cleidocranial dysplasia with bilateral posterior glenohumeral dislocation: A case-report. Orthopaedics \& Traumatology: Surgery \& Research 2015; 101:119-122.

10. KARAGÜZEL G, et al. Cleidocranial Dysplasia: A Case Report. Journal of Clinical Research in Pediatric Endocrinology 2010.2(3):134-136.

11. KOLOKITHA, OE ; IOANNIDOU,L. A 13-year-old caucasian boy with cleidocranial dysplasia: a case report. BMC Research Notes 2013; 6:6

12. MA Y; ZHAO F, YU D. Cleidocranial Dysplasia Syndrome with epilepsy: a case report. BMC pediatrics. 2019; $19: 97$.

13. MACHADO CV, et al. Características clínicas e radiográficas da displasia cleidocraniana- relato de caso. Revista da Faculdade de Odontologia UPF. 2010;15:302-6

14. PAN CY, et al. Craniofascial features of Cleidocranial Dysplasia. Journal of Dental Sciences.2017;12: 313-318.

15. PORCIUNCULA CGG, et al. Disostose cleidocraniana: relato de dois casos familiares. Radiologia Brasileira. 2013; 46(6):382-384.

16. QUEIROZ RM, et al. Displasia cleidocraniana: descrição com ênfase nos aspectos radiográficos de três casos em uma família. Medicina (Ribeirão Preto, Online.) 2017;50(6):371-6

17. SANTOS RLO, et al. Abordagem cirúrgica em paciente portador da disostose cleidocraniana. Rev. Cir. Traumatol. Buco-Maxilo-Fac., Camaragibe. 2016 v.16, n.3, p. 40-43

18. SCHNUTENHAUS S, et al. Histological examination and clinical evaluation of the jawbone of an adult patient with cleidocranial dysplasia: a case report. International Journal of Clinica \& Experimental Pathology. 2015; 8(7):85218531

19. SERRATINE ACP, ROCHA R. Displasia cleidocraniana- apresentação de um caso clínico. Arquivos Catarinenses de Médicina .2007; 36: 109-12

20. SILVA JÚNIOR AN, et al. Displasia cleidocraniana: aspectos clínicos e radiográficos e relato de um caso clínico. Revista Ciências Médicas e Biológicas, 2007; 6(1): 122-127.

21. TANAKA JL, et al. Cleidocranialdysplasia: importance of radiographic images in diagnosis of the condition. Journal of Oral Science. 2006; 48:161-6.

22. TRINDADE AKF, et al. Displasia Cleidocraniana. Revista Brasileira de Ciências da Saúde 2010; 14(2):73-76. 